\title{
Experimental Study of Two-Phase Flow in a Horizontal Circular Channel: Influence of Gas Injection on the Bubble Diameters
}

\author{
Ahmet Berk Kurtulus $^{1 \mathrm{a}}$, Nurdil Eskin ${ }^{2}$ and Emrah Deniz ${ }^{3}$ \\ 1a Graduate School of Science, Engineering and Technology, Thermo Fluids M.Sc. Program, 34496 Istanbul Technical \\ University, Turkey. \\ ${ }^{2}$ Faculty of Mechanical Engineering, 34437 Istanbul Technical University, Turkey. \\ ${ }^{3}$ Graduate School of Science, Engineering and Technology, Mechanical Engineering PhD Program, 34496 Istanbul \\ Technical University, Turkey.
}

\begin{abstract}
This study aimed to examine experimentally the effects of the injector on two-phase flow characteristics such as local void fraction and bubble diameter for water-air two- phase flow in a horizontal channel having $40 \mathrm{~mm}$ inner diameter. During the experiments, flow rate was kept constant at $3 \mathrm{l} / \mathrm{s}$ while that for air is taken as 30 and $60 \mathrm{l} / \mathrm{min}$. Measurements were made locally via dual optical probe and the results were presented.
\end{abstract}

\section{Introduction}

Multiphase flow can be considered as two-phase flow in many applications [1]. It is a complex phenomenon which is explained by tough mathematical models validated by experimental studies. Also, it is an attractive research field for the scientists to work on some of the studies performed is as follows:

Several authors have measured experimentally local void fraction and bubble size for development of twophase flow models [2-4].

Theoretical models are validated and improved via experimental results. The bubble size and its distribution are necessary in order to obtain correlations between the phases in mass, momentum and energy equations [5]. On the other hand, some physical parameters which describe phase motion play an important role in two-phase flow. Local and average void fraction and bubble size changing lead to pressure drop especially in sudden cross sectional area changes. Optimization should be considered in every stage i.e. before changing cross section or through expansion [6]. In such cases, development of analytical formulation is important. Experiments contribute to generate such analytical models [7].

Once the literature is surveyed, the majority of the experimental studies published in the literature consist of averaged values of two-phase flow parameters. In fact, one can note that many theoretical studies are validated by referring the averaged experimental values although they are constructed on separated flow model which provides the local values. Therefore, investigating twophase flow through different geometries theoretically and, validating the results by using those of experimental obtained locally is seen as a deficiency needs to be fulfilled in the literature.

In this study, characteristics of gas-liquid flow as airwater through horizontal pipe are investigated experimentally. In the experiment, the gas injected to the water flow by an immersed injector. Effects of volumetric void fraction on the two-phase flow characteristics are determined thanks to the measurements performed by dual optical probe. Radial and axial distributions of void fraction and bubble size obtained through the experiments.

\section{Method}

Schematic of the facility is shown in the figure, below.

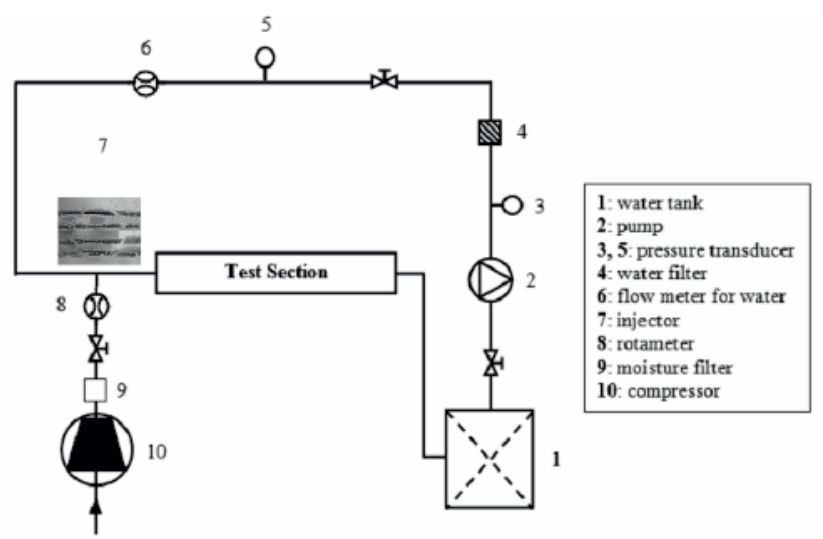

Figure 1. Schematic of the facility

\footnotetext{
${ }^{\mathrm{a}}$ Corresponding author: berkkurtulus@itu.edu.tr
} 
Water and air are chosen as the fluid couple. Water is taken from a tank by a pump at atmospheric conditions. At the exit of the pump, water is filtered with $5 \mu \mathrm{m}$ filter and sent to the electro-magnetic type flow meter to measure flow rate. The atmospheric air is compressed to 4 bar absolute pressure. At the outlet of the compressor the compressed air passes through the particle and the moisture filters. Air flow rate is controlled by a rotameter for its different values. Compressed air is then injected to the water flow by an immersed injector at where the twophase flow is formed.

Injector is designed to investigate effects of the bubble formation along the horizontal channel. The injector named as inline injector has 32 holes with $1 \mathrm{~mm}$ diameter.

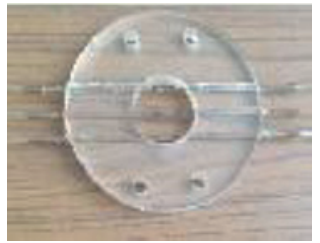

Figure 2. Inline injector

All pipes in the experimental setup are made of transparent acrylic in order to observe the flow. Test section is given as schematic in Figure 3. It consists of a pipe with prescribed lengths and diameters. Black bars stand for the measurement positions. The measurements were obtained by dual optical probe along the radial direction of the pipe at each measurement positions represented by black bars in the figure.

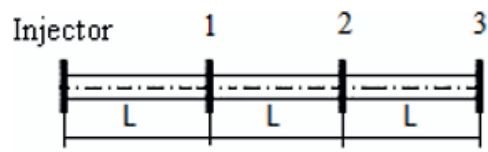

Figure 3. Schematic diagram of test section

Air-water flow through the test section is subject to the measurements by means of dual optical probe. Accuracy of the probe is given as $\pm 7 \%$ by the manufacturer company, RBI (France). Measurement principles of the optical probe can be found in [8].

Dual optical probe sinks to channel from spaces as four points that shown in Figure 4.

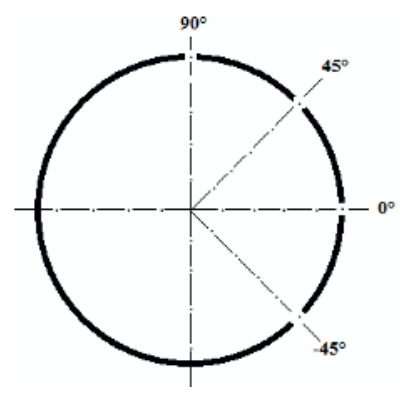

Figure 4. Measurement points via dual optical probe
Experimental data is obtained via dual optical probe at intervals $2 \mathrm{~mm}$ during 90 seconds diametrically. The measurements are taken to start from wall to entry and $\mathrm{r} / \mathrm{D}$ is made dimensionless in consequence of this method. The pipe diameter and length (L) are 40 and 330 $\mathrm{mm}$, respectively. Measurements are taken for two air flow rates, 30 and $60 \mathrm{l} / \mathrm{min}$.

\section{Results}

\subsection{Local void fraction}

During the experiments, flow rate for water is kept constant at $3 \mathrm{l} / \mathrm{s}$ while that for air is taken as 30 and 60 $1 / \mathrm{min}$. The volumetric void fraction is ratio of the flow rate of air and total volumetric flow rate of the phases. Thus volumetric void fraction $\left(\beta=\dot{Q}_{\text {air }} / \dot{Q}_{\text {total }}\right)$ of the cases are estimated to be $13.87 \%$ and $25.31 \%$, respectively. Experimental values for radial distribution of local void fraction along the test section at different flow rates are plotted below.

Local void fraction distributions measured at 90 degree position for two volumetric void fraction values are given in Figure 5.
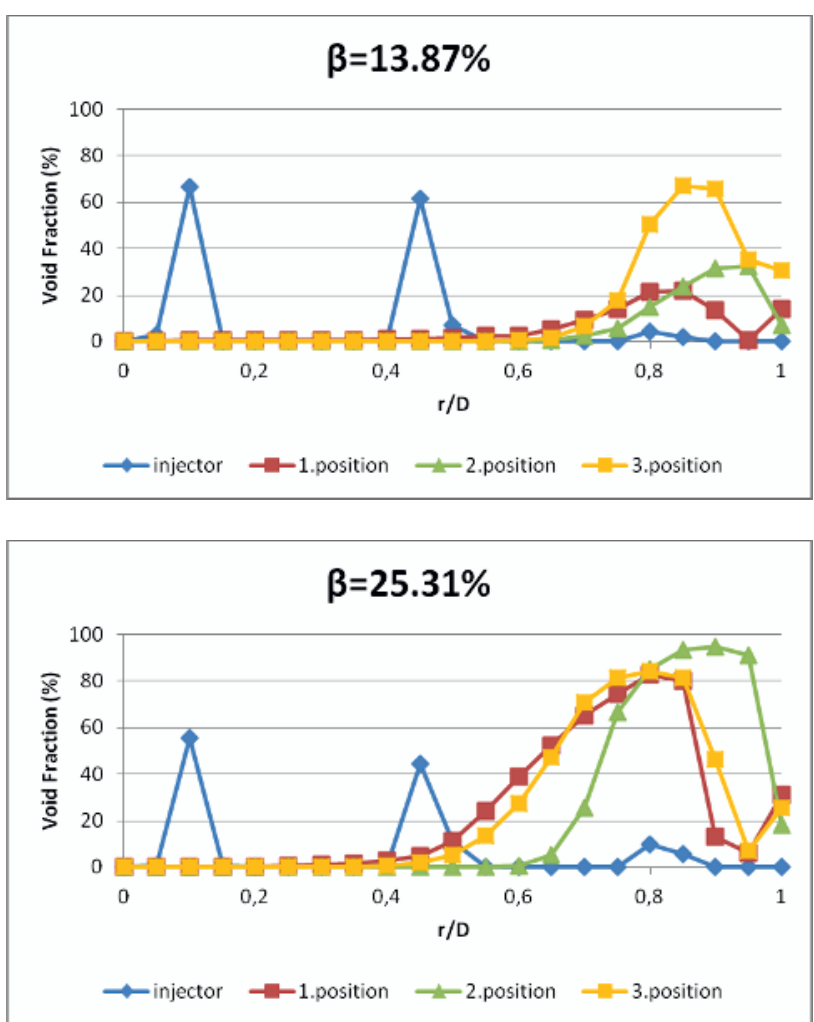

Figure 5. Local void fraction distribution at $90^{\circ}$

At the injector outlet, the local void fraction values along the diameter shows similar distributions at two different air flow rates. As the injector effect vanishes, void fraction values gets higher values especially at the upper part of the pipe.

Local void fraction distributions at different gas flow rates obtained at the 45 degree measurements are given in Figure 6. 

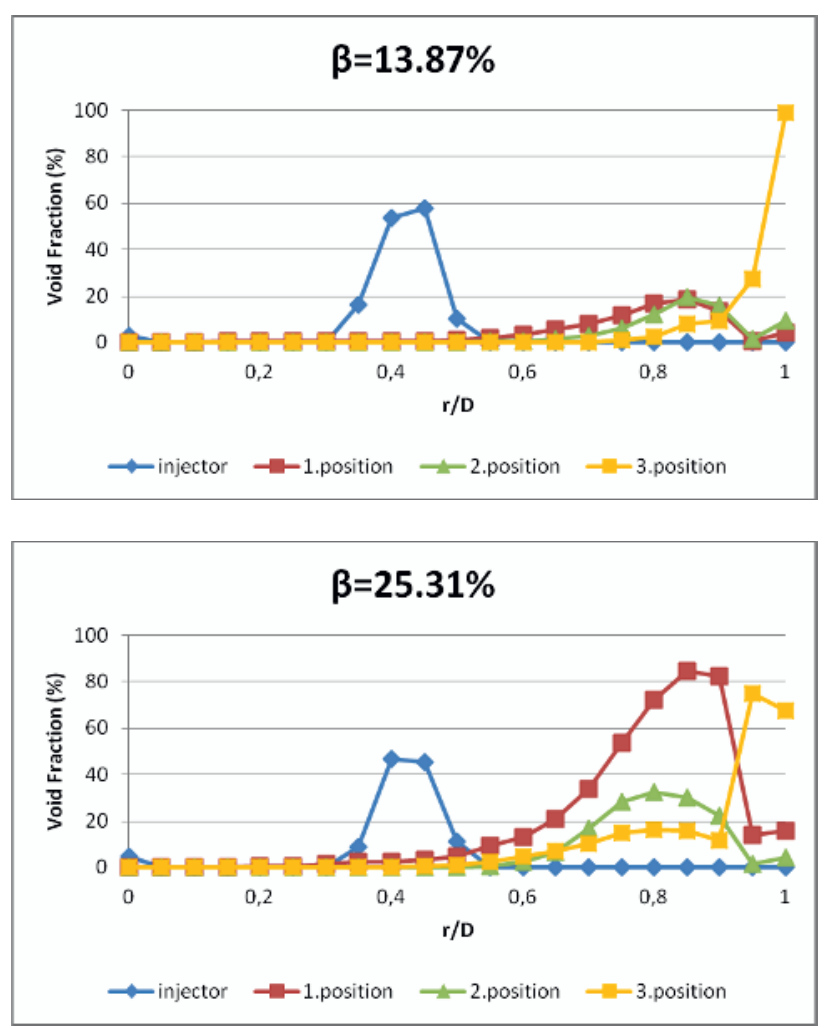

Figure 6. Local void fraction distributions at $45^{\circ}$

As it is easily observed from the figures that, as the flow rate increases, the measured local void fraction values are almost the same values at the injector outlet. At the first position of the test section, the local void fraction values show significant increase due to the influence of the buoyancy force on the bubble movement along the pipe.
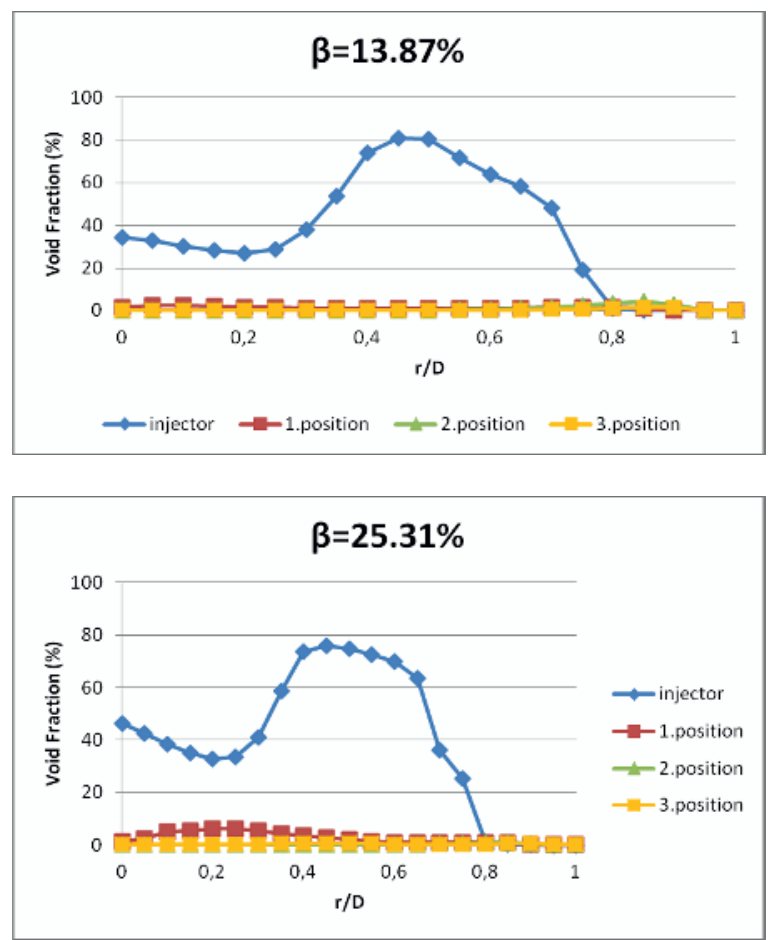

Figure 7. Local void fraction distributions at 0 degree
The local void fraction values get higher values and show a distribution at the injector outlet in this section of the pipe (Figure 7). It is appeared that the bubbles gather in the middle of the pipe rather than wall of the pipe.

Local void fraction distribution at the -45 degree is given in Figure 8 at two volumetric void fraction values.
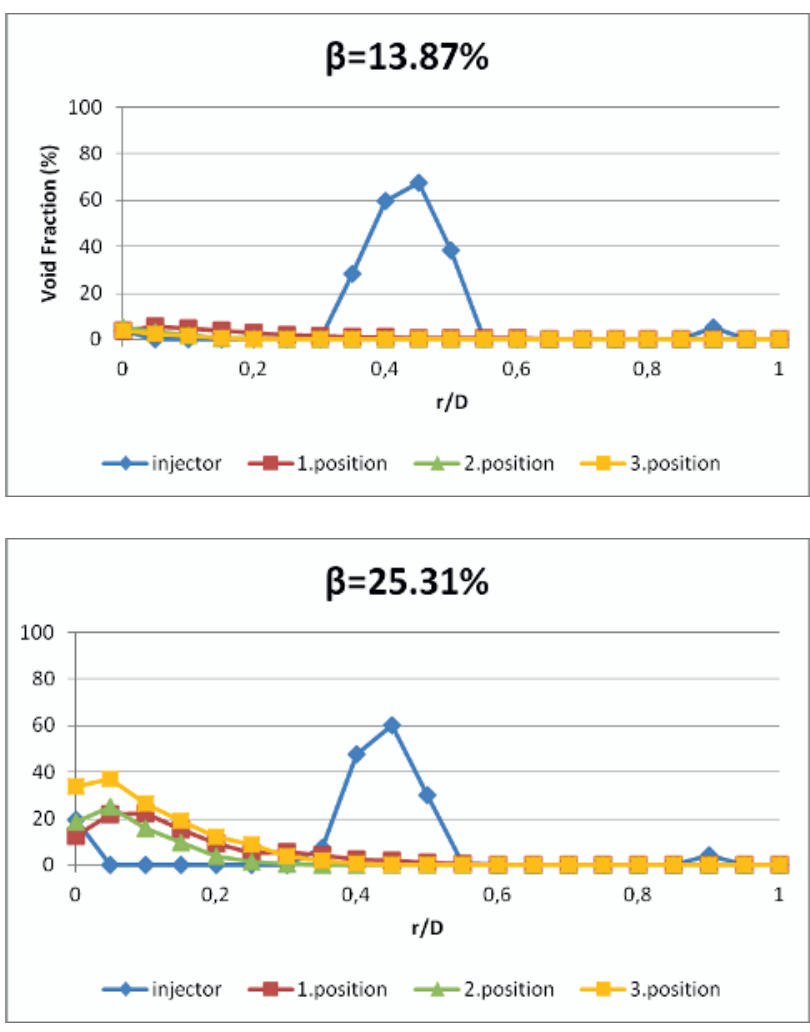

Figure 8. Local void fraction distributions at $-45^{\circ}$

This measurement section refers to the bottom of the pipe therefore void fraction values are between 0.02-0.38 at all positions except the injector outlet. The bubbles gather upper part due to the buoyancy force and it can be stated that the local void fraction values increase with the increase in air flow rate.

\subsection{Bubble sizes}

Bubble size is also measured during the experiments and it is observed that the air flow rate has an important effect on the size variations.

Due to momentum of the flow and forces between the phases, injected bubbles break up or coalesce and, cause a variation in bubble diameter along the flow. Measured values of bubble size at four radial direction along the pipe are shown in Figure 9-11 for inline injector at two volumetric void fraction values.

In all measurements, it is observed that the measured bubble diameter values at the injector outlet decrease as the flow rate reaches higher values due to the effect of the injector. Bubble size values get bigger ones due to coalesce at the upper part of the pipe at all measurement positions. 

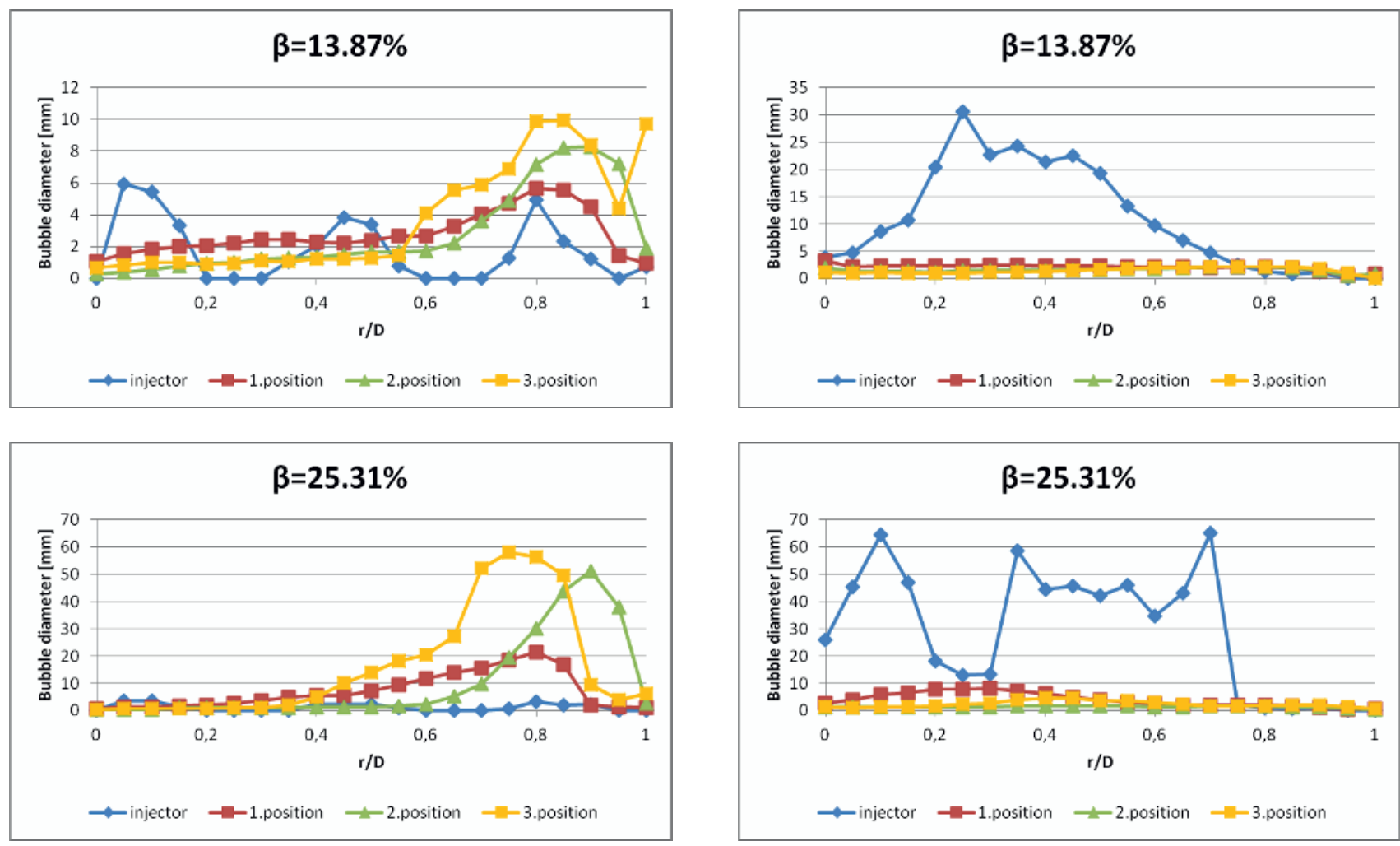

Figure 9. Bubble diameter profiles at $90^{\circ}$

Figure 11. Bubble diameter profiles at $0^{\circ}$
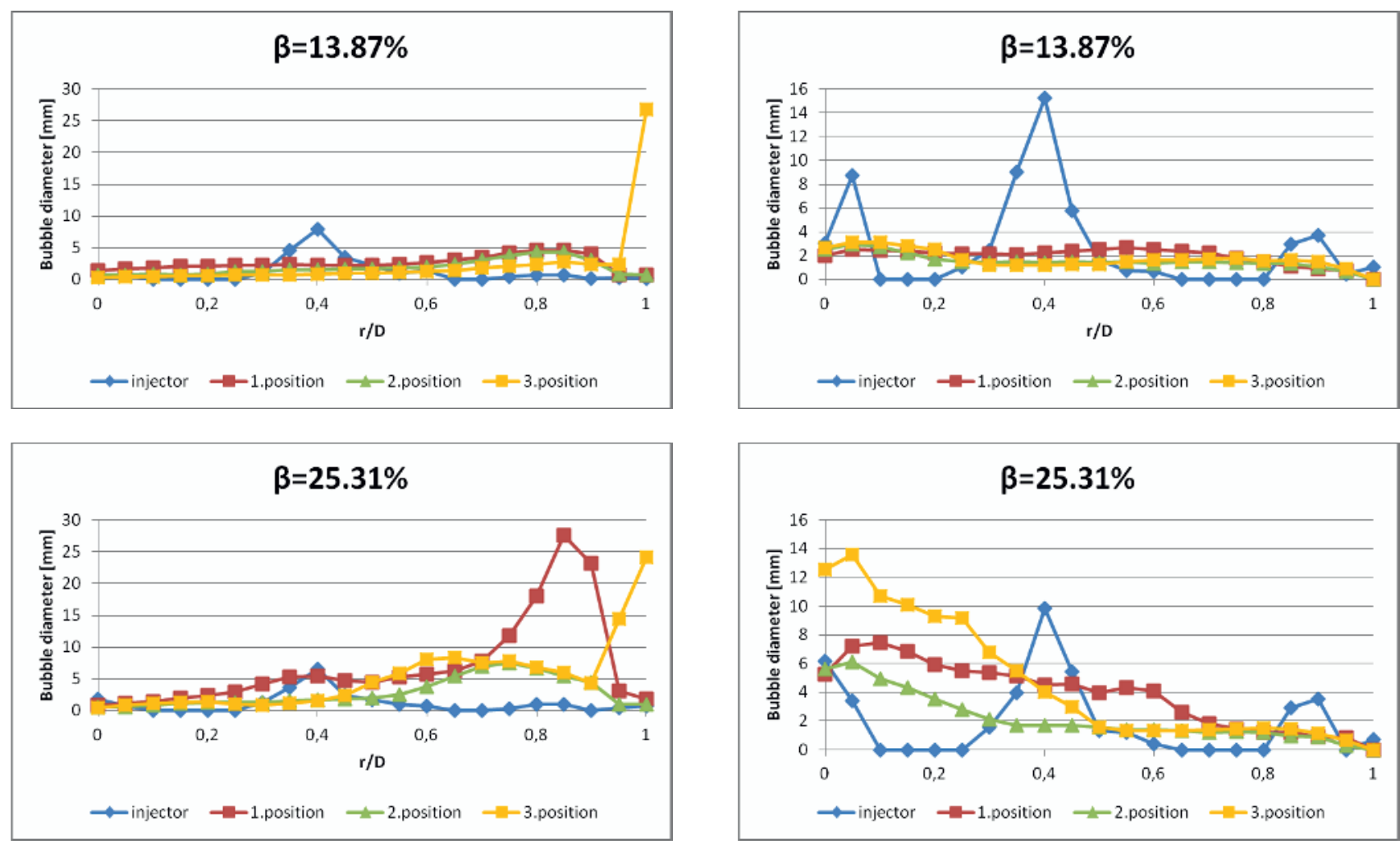

Figure 10. Bubble diameter profiles at $45^{\circ}$

Figure 12. Bubble diameter profiles at $-45^{\circ}$ 
Bubble sizes are around 5 to $10 \mathrm{~mm}$ at the outlet of the injection, as the flow rate increases the bubble diameters gets larger and reaches $60 \mathrm{~mm}$ in diameter. These values observed especially in the first measurement position. The bubble size profile is consistency with the void fraction distributions as expected. Through the measurements the hydrodynamic parameters of the air-water two phase flow can be obtained at axial and radial direction of the pipe.

\section{Conclusion}

In this study, local void fraction distribution and bubble sizes profiles of air-water two phase flow through horizontal pipe is investigated experimentally. Local void fraction increases with increasing value of volumetric void fraction. Void fraction and bubble size are varying relatively. Bubble size values are getting larger with local void fraction distribution for all cases considered, as expected.

It is apparent that bubbles gather due to the influence of buoyancy force at the upper part of the pipe. Hence, the bubble size profile and local void fraction increase in specified tendency from injector to third position. The results are contributed to correlate between local void fraction values and bubble diameter.

\section{References}

1. G.B. Wallis, One-dimensional Two-phase Flow, (McGraw Hill 1969).

2. M. Bonizzi and R.I. Issa, Int. J. of Multiphase Flow, 29, 1685 (2003).

3. M.A. Rahman, M. Balzan, T. Heidrick and B.A. Fleck, Int. J. of Multiphase Flow, 38, 35 (2012).

4.K. Yoneda, A. Yasuo and T. Okawa, Nuclear Eng. and Des., 217, 267 (2002).

5. R.H.S. Winterton and J.S. Munaweera, Che. Eng. and Processing, 40, 437 (2001).

6. F. Aloui, L. Dobliez, J. Legrand, M. Souhar, Exp. Thermal and Fluid Sci., 19, 118 (1999).

7. W.H. Ahmet, C.Y. Ching, M. Shoukri, Int. J. of Multiphase Flow, 33, 575 (2007).

8. F. François, J. Garnier and G. Cubizolles, Measurement Science and Technology, 14, 929 (2003). 